Discrete Comput Geom 29:445-457 (2003)

DOI: $10.1007 /$ s00454-002-2836-y

\title{
Doubly Transitive Automorphism Groups of Combinatorial Surfaces
}

\author{
W. Kimmerle and E. Kouzoudi \\ Mathematisches Institut B, Universität Stuttgart, \\ D-70550 Stuttgart, Germany \\ kimmerle@mathematik.uni-stuttgart.de \\ evgenia.kouzoudi@bw-bank.de
}

\begin{abstract}
The combinatorial surfaces with doubly transitive automorphism groups are classified. This is established by classifying the automorphism groups of combinatorial surfaces which act doubly transitively on the vertices of the surface. The doubly transitive automorphism groups of combinatorial surfaces are the symmetric group $S_{4}$, the alternating group $A_{5}$ and the Frobenius group $C_{7} \cdot C_{6}$. In each case the combinatorial surface is uniquely determined. The symmetric group $S_{4}$ acts doubly transitively on the tetrahedron surface, the alternating group $A_{5}$ on the triangulation of the projective plane with six vertices and the Frobenius group $C_{7} \cdot C_{6}$ on the Moebius torus with seven vertices.
\end{abstract}

\section{Statement and Discussion of the Result}

The object of this note is the classification of the automorphism groups of combinatorial surfaces which act doubly transitively on the vertices of the surface. For brevity we call such a group a doubly transitive automorphism group of a combinatorial surface. Combinatorial surfaces with doubly transitive automorphism groups may be regarded like the regular (reflexible and irreflexible) maps of Coxeter and Moser [1] as non-spherical analogues of the Platonic solids. We show that there are only three such surfaces.

Theorem. The doubly transitive automorphism groups of combinatorial surfaces are the symmetric group $S_{4}$, the alternating group $A_{5}$ and the Frobenius group $C_{7} \cdot C_{6}$. In each case the combinatorial surface is uniquely determined. The symmetric group $S_{4}$ acts doubly transitively on the tetrahedron surface, the alternating group $A_{5}$ on the triangulation of the projective plane with six vertices and the Frobenius group $C_{7} \cdot C_{6}$ on the Moebius torus with seven vertices. 
The method of the proof is mainly group-theoretical and involves standard results on soluble doubly transitive groups and on Zassenhaus groups.

This article was motivated by the thesis of Lutz [9]. Extending computations of Kühnel and Lassmann [8] he classified all combinatorial manifolds with less than 14 vertices and a vertex transitive automorphism group. These manifolds are of dimension $2 \leq d \leq 11$. Moreover, Lutz showed that there are exactly 77 combinatorial surfaces on $n \leq 1 \overline{5}$ vertices which have a vertex transitive automorphism group. Also all transitive permutation groups of degree less than 16 which occur as vertex transitive automorphism group of a combinatorial manifold have been classified in [9]. Note that not all transitive permutation groups occur. It is natural to impose additional properties on the automorphism group of the combinatorial manifold. The above theorem answers completely the first obvious case which may be studied in this context.

\section{Notations and Preliminaries}

Definition 2.1. A combinatorial surface $S$ is a two-dimensional pure simplicial complex with the following properties:

(a) Each edge is contained in precisely two triangles.

(b) $S$ is connected

(c) The link of each vertex is a cycle, i.e. a triangulated 1-sphere.

Remark 2.2. (a) Let $\mathcal{K}$ be a simplicial complex and $s \in \mathcal{K}$. Then the link of $s$ is defined as

$$
\operatorname{link}(s):=\{t \in \mathcal{K} \mid t \cap s=\emptyset \text { and } t \cup s \in \mathcal{K}\}
$$

(b) Connectedness is defined as usual, i.e. any two vertices are connected by a sequence of edges.

(c) Condition 2.1(a) is called the pseudo-manifold property.

(d) Each combinatorial surface is, in the terminology of [10], an abstract 3-polytope. In order to get the appropriate underlying poset of an abstract polytope one has just to add a least face $F_{-1}$ and a greatest face $F_{3}$. Note that property $2.1(\mathrm{c})$ is not part of the definition of an abstract polytope.

Lemma 2.3. Let $G$ be a doubly transitive automorphism group of a combinatorial surface with $n$ vertices. Then either $|G|=n \cdot(n-1) \cdot 2$ or $|G|=n \cdot(n-1)$.

Proof. As $G$ acts doubly transitively on the vertices, the link of a vertex is an $(n-1)$ cycle. Each automorphism fixing a vertex induces an automorphism of the link of the vertex. The automorphism group of an $(n-1)$-cycle is a dihedral group of order $2(n-1)$. Since $G$ is doubly transitive on the $n$ vertices, its order is divisible by $n \cdot(n-1)$.

Corollary 2.4. The symmetric group $S_{4}$ is the only threefold transitive automorphism group of a combinatorial surface. 
Proof. Let $G$ be such a group acting on $n$ vertices. As $G$ is threefold transitive, $|G|$ is divisible by $n \cdot(n-1) \cdot(n-2)$. Lemma 2.3 shows that the only possibilities are $|G|=24, n=4$ or $|G|=6, n=3$. However, a triangle is not a combinatorial manifold. Thus the first case remains and $G=S_{4}$ follows.

Lemma 2.5. Let $S$ be a combinatorial surface with $n$ vertices, $k$ edges and $d$ triangles. Assume that the automorphism group of $S$ acts doubly transitively on the vertices. Then

(i) $k \cdot 2=d \cdot 3$,

(ii) $k=\frac{1}{2} \cdot n \cdot(n-1)$,

(iii) $d=\frac{1}{3} \cdot n \cdot(n-1)$ and

(iv) 3 divides either $n$ or $n-1$.

Proof. For the proof of (i) observe that each edge is contained in precisely two triangles and each triangle has three edges.

Since $G$ is doubly transitive, each vertex is connected with each other vertex by an edge. This shows (ii). Part (iii) is a consequence of (i) and (ii). Part (iv) follows from (iii).

Lemma 2.6. Let $G$ be a doubly transitive automorphism group of a combinatorial surface $S$ with $n$ vertices.

(i) If $|G|=n \cdot(n-1)$, then the stabilizer of an edge is of order 2. The stabilizer of a triangle has order 3 .

(ii) If $|G|=2 \cdot n \cdot(n-1)$, then the stabilizer of an edge has order 4 . The stabilizer of a triangle has order 6 .

(iii) The group $G$ acts transitively on the set of triangles of $S$.

Proof. We first prove part (iii). Suppose that the triangles of the surface are a union of more than two orbits. Then there are three orbits with representatives

$$
\left\{1, i_{1}, j_{1}\right\}, \quad\left\{1, i_{2}, j_{2}\right\}, \quad\left\{1, i_{3}, j_{3}\right\} .
$$

As $G$ is doubly transitive, there are elements $e_{1}, e_{2} \in G$ with

$$
e_{1}\left(\left\{1, i_{1}\right\}\right)=\left\{i_{3}, j_{3}\right\} \quad \text { and } \quad e_{2}\left(\left\{1, i_{2}\right\}\right)=\left\{i_{3}, j_{3}\right\}
$$

Consequently, $e_{1}\left(\left\{1, i_{1}, j_{1}\right\}\right)=\left\{i_{3}, j_{3}, x_{1}\right\}$ and $e_{2}\left(\left\{1, i_{2}, j_{2}\right\}\right)=\left\{i_{3}, j_{3}, x_{2}\right\}$. This means that the edge $\left\{i_{3}, j_{3}\right\}$ occurs in each of the three orbits in contradiction to the assumption that an edge is only contained in two triangles.

Suppose now that there are precisely two orbits $B_{1}, B_{2}$ of triangles with representatives

$$
\{1, a, b\} \in B_{1} \quad \text { and } \quad\{1, c, d\} \in B_{2} .
$$

As $G$ is doubly transitive, there are elements $e_{3}, e_{4} \in G$ with $e_{3}(\{1, a\})=\{1, c\}$ and $e_{4}(\{1, c\})=\{1, a\}$. Thus there exist triangles of the form $\{1, c, x\} \in B_{1}$ and $\{1, a, y\} \in$ $B_{2}$. It follows that each edge occurs in each orbit in precisely one triangle. 
Case $A:|G|=2 \cdot n(n-1)$. Then the stabilizer stab(1) has order $2 \cdot(n-1)$. Thus it contains all rotations and reflections of link(1).

Since $G$ is doubly transitive, all edges of the form $\{1, i\}, i \in\{2, \ldots, n\}$, occur in the combinatorial surface $\mathcal{K}$. Thus for $i \in\{2, \ldots, n\}$ the vertex $i$ is an element of link(1). Since $G$ contains all reflections of $\operatorname{link}(1)$, there exists for each $i \in\{2, \ldots, n\}$ a reflection $e_{i}=(1)(i) \cdots \in \operatorname{stab}(1)$. Hence $e_{i}(\{1, i, j\})=\{1, i, k\}$ is a triangle of $S$ and the edge $\{1, i\}$ occurs in the orbit of $\{1, i, j\}$ in two triangles. This contradicts the assumption that there are two orbits of triangles.

Case $B:|G|=n \cdot(n-1)$. Suppose that stab(1) consists only of rotations. Then all rotations of link(1) belong to $\operatorname{stab}(1)$. Let $\{1, a, b\}$ be a triangle of $S$. Then there exists a rotation $e=(1)(a, b, c, \ldots, z) \in \operatorname{stab}(1)$ and we get $e(\{1, a, b\})=\{1, b, c\}$. Consequently, the edge $\{1, b\}$ occurs twice in the same orbit, a contradiction.

Suppose that stab(1) consists of rotations and reflections. The stabilizer stab(1) acts transitively on the vertices $\{2, \ldots, n\}$. Thus the reflections in stab(1) fix no vertex of $\operatorname{link}(1)$. Let $e=(1)(i, j) \cdots$ be such a reflection. The edge $\{i, j\}$ occurs in $S$ in precisely two triangles:

$$
\{i, j, k\} \text { and }\{i, j, l\} .
$$

We may assume that $k \neq 1$. Then

$$
e(\{i, j, k\})=\left\{i, j, k^{\prime}\right\} \quad \text { with } \quad k \neq k^{\prime}
$$

because 1 is the only vertex fixed by $e$. Hence $\{i, j\}$ occurs twice in a triangle orbit, contradiction.

Thus the triangles of the combinatorial surface form a single orbit and (iii) is established.

For parts (i) and (ii) note that by Lemma 2.5 the total number of triangles is $d=$ $\frac{1}{3} \cdot n \cdot(n-1)$. As all the triangles are in one orbit, the order of the stabilizer of a triangle is given by $|G| / d$. Since $G$ is doubly transitive on the vertices, it acts transitively on the edges. So the order of the stabilizer of an edge is $|G| / k$ and $k$ is given by Lemma 2.5(ii).

Remark 2.7. Let $S$ be a combinatorial surface and assume that its automorphism group $G$ acts doubly transitively on the vertices of $S$. Then Lemma 2.6 shows that $S$ is a regular abstract 3-polytope in the case where $|G|=n \cdot(n-1) \cdot 2$, see Sections 2A and 2B of [10]. In the other case $S$ is a chiral abstract 3-polytope. We call a combinatorial surface $S$ regular provided it is a regular abstract 3-polytope, and $S$ is called chiral in the case where it is a chiral abstract 3-polytope.

Corollary 2.8. Let $G \leq S_{n}$ acting doubly transitively on the set of vertices $\{1, \ldots, n\}$ of a combinatorial surface $S$. Then, if $\{a, b, c\}$ is a triangle of $S$, there exists $g \in G$ of the form $(a, b, c), \ldots$.

Proof. By Lemma 2.6 the order of the stabilizer of a triangle is divisible by 3 . 
Lemma 2.9. Let $S$ be a combinatorial surface with $n$ vertices and the doubly transitive automorphism group $G$.

(a) If $S$ is chiral and $n-1$ is odd, then each non-trivial element of $G$ fixes at most one vertex. $G$ is a soluble Frobenius group and has degree $2^{f}$.

(b) If $S$ is regular and $n-1$ is odd, then the non-trivial permutations of $G$ fix at most two vertices. $G$ is a Zassenhaus group or is isomorphic to $\Gamma L$ over a field of $2^{p}$ elements, $p$ a prime, and has degree $2^{p}$.

Proof. Assume that $g \in G$ fixes two vertices $i$ and $j$. Since $G$ acts doubly transitively, the vertices $i$ and $j$ are connected by an edge. $g$ induces on link $(i)$ a reflection or the identity.

If $S$ is chiral and $n-1$ is odd, then the stabilizer of a vertex has odd order and $g$ must be the identity. Consequently, the non-trivial elements of $G$ fix at most one vertex. Thus $G$ is a Frobenius group [4, V, 8.2]. As the stabilizer of a vertex is cyclic, the Frobenius complements of $G$ are cyclic. By a well known theorem of J. Thompson the Frobenius kernel is nilpotent [4, V, 8.7a)]. Thus $G$ is soluble. Soluble primitive permutation groups - so, in particular, doubly transitive soluble groups-are of prime power degree [4, II, 3.2]. Hence $G$ has degree $2^{f}$.

If $S$ is regular and $n-1$ is odd, a reflection of the link of a vertex fixes precisely one vertex. Then non-identity elements of $G$ fix at most two vertices and $G$ has an element which fixes two vertices. If $G$ has no regular normal subgroup, then $G$ satisfies the definition a Zassenhaus group [5, XI, 1.2]. In the other case the structure of $G$ follows from a theorem of Feit [2], [5, XI, 1.1].

Example 2.10. The symmetric group $S_{4}$ acting on the tetrahedron gives an example of a regular combinatorial surface with an even number $n$ of vertices. We shall see in Section 3 that there are no chiral combinatorial surfaces with a doubly transitive automorphism group and even $n$.

Lemma 2.11. Let $S$ be a combinatorial surface with $n$ vertices and the doubly transitive automorphism group $G$.

(a) If $S$ is chiral and $n-1$ is even, then each non-trivial element of $G$ fixes at most one vertex. $G$ is a Frobenius group and has a regular normal subgroup.

(b) The surface $S$ cannot be regular in the case where $n-1$ is even.

Proof. (a) Suppose that $S$ is chiral and that $(n-1)$ is even. Assume that the stabilizer $\operatorname{stab}(i)$ of a vertex $i$ contains reflections of $\operatorname{link}(i)$. As $S$ is chiral, the stabilizer stab(1) has order $(n-1)$ and acts transitively on link(1). Hence no reflection of stab(1) fixes a vertex. Clearly, rotations of stab(1) (except the trivial one) do not fix a vertex of the link. Thus $G$ has no non-trivial element fixing more than one vertex. It follows that $G$ is a Frobenius group [4, V, 8.2]. The stabilizer of a vertex has order $(n-1)$ and is a subgroup of the automorphism group of an $(n-1)$-cycle. Since this automorphism group is a dihedral group of order $2 \cdot(n-1)$, the stabilizer of a vertex is soluble. Consequently, $G$ 
has soluble Frobenius complements. As in the proof of Lemma 2.9 it now follows that $G$ has a regular normal subgroup of prime power order.

(b) Suppose now that $n-1$ is even and that $S$ is regular. Then $G$ has non-trivial elements which fix at least three vertices of the surface.

As $|G|=2 \cdot n \cdot(n-1)$, the stabilizer of a vertex $a$ is the full automorphism group of an $(n-1)$-cycle, i.e. it is a dihedral group of order $2 \cdot(n-1)$ consisting of $n-1$ rotations and $n-1$ reflections. Since $n-1$ is even, $(n-1) / 2$ reflections fix precisely two vertices of $\operatorname{link}(a)$ and $(n-1) / 2$ reflections fix no vertex of $\operatorname{link}(a)$.

Any two vertices of $S$ are joined by an edge. Thus for each vertex $a$ of $S$ and for each $b \in \operatorname{link}(a)$ there is a reflection $r(a, b) \in G$ which fixes $a, b$ and a uniquely determined third vertex $\rho(a, b) \in \operatorname{link}(a)$. There is no other group element which fixes $a, b$ and $\rho(a, b)$ because there is only one reflection of the link of $a$ fixing $b$. This argument also applied with respect to $\operatorname{link}(b)$ and $\operatorname{link}(\rho(a, b))$ yields

$$
r(a, b)=r(b, a)=r(b, \rho(a, b))=r(\rho(a, b), b)=r(\rho(a, b), a)=r(a, \rho(a, b)) .
$$

If $a^{\prime}, b^{\prime}$ are vertices with $\left|\left\{a^{\prime}, b^{\prime}, \rho\left(a^{\prime}, b^{\prime}\right)\right\} \cap\{a, b, \rho(a, b)\}\right| \leq 1$, then $r(a, b) \neq r\left(a^{\prime}, b^{\prime}\right)$ because no group element of $G$ fixes more than three vertices except the identity. Consequently, we get $\frac{1}{6} \cdot n \cdot(n-1)$ different group elements which fix precisely three vertices.

For $g \in G$ denote by $\delta(g)$ the number of 2-cycles which occur in the cycle decomposition of $g$. By 20.5(a) in Chapter V of [4]

$$
\sum_{g \in G} \delta(g)=\frac{1}{2} \cdot|G|
$$

because $G$ is doubly transitive.

If $g \in G$ fixes precisely three vertices, then $g$ is a reflection of the link of one of the fixed vertices. Therefore $\delta(g)=(n-3) / 2$. By the previous argument there are $n \cdot(n-1) \cdot \frac{1}{6}$ elements of $G$ which fix precisely three vertices.

For each vertex $a$ the group $G$ has $(n-1) / 2$ reflections which fix precisely $a$. All rotations of link $(a)$ except the trivial one fix also only the vertex $a$. Denote by $D$ the group of rotations of link $(a)$. Then $D=\langle d\rangle$ and $d$ consists of one $(n-1)$-cycle. Consequently, $d^{(n-1) / 2}$ is the only element in $C$ with 2-cycles in its cycle decomposition.

Thus, if $g \in G$ fixes precisely one vertex $a$ and if 2-cycles occur in the cycle decomposition of $g$, then $g$ is a reflection of $\operatorname{link}(a)$ fixing no element of this link or $g$ is a rotation of $\operatorname{link}(a)$ of order 2 . Clearly, $\delta(g)=(n-1) / 2$ and there are $n \cdot((n-1) / 2+1)$ such elements of $G$. Therefore

$$
n \cdot \frac{(n-1)}{6} \cdot \frac{(n-3)}{2}+n \cdot\left(\frac{(n-1)}{2}+1\right) \cdot \frac{(n-1)}{2} \leq \sum_{g \in G} \delta(g)=\frac{|G|}{2}=n \cdot(n-1) .
$$

It follows that $n \leq 3$. However, there is no combinatorial surface with three vertices. This completes the proof.

We thank the referee for pointing out an error in a previous version of Lemma 2.11(b). 


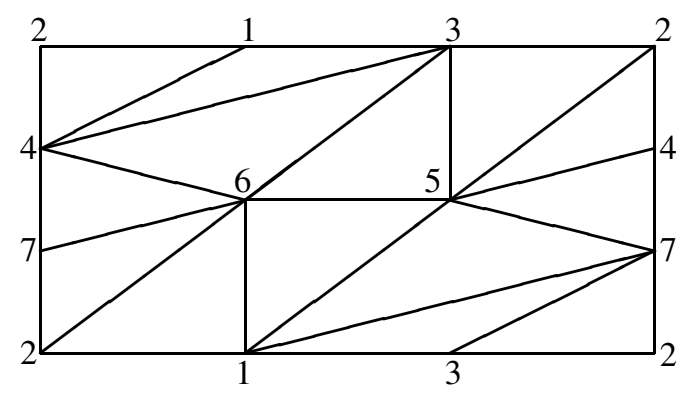

Fig. 1. The Moebius torus, $\{3,6\}_{2,1}$ in Coxeter's notation.

Example 2.12. The Moebius torus (see Fig. 1) is a chiral combinatorial surface with a doubly transitive automorphism group isomorphic to the Frobenius group $C_{7} \cdot C_{6}$.

\section{The Soluble Case}

If $G$ is a doubly transitive group with a regular normal subgroup, then $G$ has prime power degree [4, II, 2.3a)]. In particular, this is the case when $G$ is soluble [4, II, 3.2]. Moreover, it turns out that if $G$ is a doubly transitive automorphism group of a combinatorial surface with a regular normal subgroup, then $G$ is soluble.

In this section we prove the following.

Proposition 3.1. The only doubly transitive automorphism groups of combinatorial surfaces with a regular normal subgroup of order $q=p^{f}$ are the symmetric group $S_{4}$ and the Frobenius group $C_{7} \cdot C_{6}$.

For the proof we need the following elementary facts on permutation groups.

Lemma 3.2. Let $N$ be a regular and abelian permutation group.

(i) Assume that $n=(i, \ldots, j, \ldots, k, \ldots) \cdots \in N$. Then there is no element in $N$ of the form $g=(i, \ldots, j, \ldots)(k, \ldots) \cdots$.

(ii) Assume that $n_{1}=\left(a_{1}, a_{2}, \ldots, a_{p}\right)\left(b_{1}, b_{2}, \ldots, b_{p}\right) \cdots \in N$ and that $n_{2}=$ $\left(a_{1}, b_{1}, \ldots\right) \cdots \in N$. Then

$$
n_{2}=\left(a_{1}, b_{1}, \ldots\right)\left(a_{2}, b_{2}, \ldots\right)\left(a_{3}, b_{3}, \ldots\right) \cdots
$$

Proof. (i) Suppose $g=(i, \ldots, j, \ldots)(k, \ldots) \in N$. Then $g^{m}=(i, j, \ldots)(k, \ldots) \cdots$ for some $m \in \mathbb{N}$ and $n^{l}=(i, j, \ldots, k, \ldots)$ for some $l \in \mathbb{N}$. Clearly, $g^{m} \neq n^{l}$. On the other hand, as $N$ is regular, $n^{l} \in N$ is the unique element mapping $i$ to $j$. Thus $g^{m}=n$, contradicting the assumption $g \in N$. 
(ii) Since $N$ is abelian, we get

$$
n_{2} \circ n_{1}=\left(a_{1}, b_{2}, \ldots\right) \cdots=n_{1} \circ n_{2}
$$

Consequently, $n_{2}$ maps $a_{2}$ to $b_{2}$. The cycle of $n_{2}$ containing $a_{2}$ and $b_{2}$ does not contain $a_{1}$. For, if $a_{1}$ and $a_{2}$ are in the same cycle, then by part (i) $b_{2}$ cannot be in this cycle. Thus

$$
n_{2}=\left(a_{1}, b_{1}, c_{1}, \ldots\right)\left(a_{2}, b_{2}, \ldots\right) \cdots
$$

Calculating $n_{1} \circ n_{2}$ again we see analogously that $n_{2}$ maps $a_{3}$ to $b_{3}$ and that $n_{2}$ is of the stated form.

Proof of Proposition 3.1. Denote the vertices of the surface by $\{1, \ldots, q\}$. Clearly, $\operatorname{link}(q)$ is a $(q-1)$-cycle. Let $\Delta=\left\{1, a_{1}, q\right\}$ be a triangle with $a_{1} \in\{2, \ldots, q-1\}$. Then the edge $\left\{1, a_{1}\right\}$ belongs to $\operatorname{link}(q)$. As $\operatorname{stab}(q)$ acts transitively on $\{1, \ldots, q-1\}$, there exists $g \in \operatorname{stab}(q)$ of the form

$$
g=\left(1, a_{1}, \ldots\right)(q) \cdots
$$

The group element $g$ is either a rotation or a reflection. We show that we can always find a rotation with this property.

Suppose that $g$ is a reflection. Then $g=\left(1, a_{1}\right) \cdots(q)$. Clearly, $g\left(\left\{1, a_{1}, q\right\}\right)=$ $\left\{1, a_{1}, q\right\}$ and $g \in \operatorname{stab}\left(\left\{1, a_{1}, q\right\}\right)$. By Lemma 2.6 we know that $\operatorname{stab}\left(\left\{1, a_{1}, q\right\}\right)$ has either three or six elements. Since $g$ has order 2 , we get that $\left|\operatorname{stab}\left(\left\{1, a_{1}, q\right\}\right)\right|=6$ and $|G|=2 \cdot q \cdot(q-1)$. Thus $\operatorname{stab}(q)$ contains all rotations of $\operatorname{link}(q)$. Since 1 and $a_{1}$ are connected by an edge, we get a rotation mapping 1 to $a_{1}$. Thus we may assume that there is a rotation $h$ of the form

$$
h=\left(1, a_{1}, a_{2}, \ldots, a_{k}\right)(q) \quad \text { with } \quad k=q-2 .
$$

The triangles with edge $\{1, q\}$ are therefore $\left\{1, a_{1}, q\right\}$ and $\left\{1, a_{k}, q\right\}$.

Let $N$ be the regular normal subgroup of $G$. Denote by $\Omega$ the set of vertices of the surface. Let $i \in \Omega$. Since $G$ is doubly transitive on $\Omega$, it follows that $N$ acts transitively on $\Omega \backslash\{i\}$. The action of $\operatorname{stab}(i)$ on $\Omega \backslash\{i\}$ is similar to that of $\operatorname{stab}(i)$ on $N \backslash\left\{1_{G}\right\}$ given by conjugation [4, II, 2.2]. Consequently, $N$ is a minimal normal subgroup and $N$ is elementary abelian [4, II, 2.3(a)]. From a result of Galois it follows that $N$ is the unique minimal normal subgroup of $G$ [4, II, 3.2].

Let $e$ be the unique element of $N$ with $e(1)=q$. Next we discuss the possibilities in which way $1, a_{1}, a_{k}, q$ occur in $e$. Put

$$
e(q)=x, \quad e\left(a_{1}\right)=y, \quad e\left(a_{k}\right)=z
$$

Then

$$
e\left(\left\{1, a_{1}, q\right\}\right)=\{q, y, x\} \quad \text { and } \quad e\left(\left\{1, a_{k}, q\right\}\right)=\{q, z, x\} .
$$

This means that $y$ and $x$ and, on the other hand, $x$ and $z$ are neighbours in $h$. As the 
vertices are ordered in $h$ in the same way as they are connected by an edge in $\operatorname{link}(q)$, we get two possibilities for $h$ :

$$
h=h_{1}=\left(1, a_{1}, \ldots, y, x, z, \ldots, a_{k}\right) \quad \text { or } \quad h=h_{2}=\left(1, a_{1}, \ldots, z, x, y, \ldots, a_{k}\right) .
$$

Suppose that $h=h_{2}$. Then $e_{2}=h_{2} \circ e \circ h_{2}^{-1} \in N$ maps $a_{1}$ to $q$ and $q$ to $y . f_{2}=h_{2}^{-1} \circ e \circ h_{2}$ maps $a_{k}$ to $q$ and $q$ to $z$. As $e_{2}(1)=f_{2}(1)=x$, it follows that $e_{2}=f_{2}$ and we get the contradiction that $a_{1}=a_{k}$. Consequently, $h=h_{1}$. Then $e_{1}=h \circ e \circ h^{-1}$ maps $a_{1}$ to $q$ and $q$ to $z$. By Lemma 3.2(i) we get the following cases:

Case 1. $a_{1}, a_{k}, z$ and $y$ are either in the same cycle of $e$ as $1, q$ and $x$

or

Case 2. 1, $a_{1}$ and $a_{k}$ are in pairwise different cycles of $e$.

Next we consider the special cases when $y$ or $z$ coincide with at least one of $a_{1}, a_{k}$ or 1 . Note that this can only happen in case 1 .

$a_{1}=y$ or $a_{k}=z$ is impossible because $e$ has no fixed points. If $1=y$, then looking at $h_{1}$ we see that $a_{1}=x$. Thus $e=\left(1, q, a_{1}\right) \cdots$. Hence $a_{k}$ is in another cycle of $e$. Similarly, if $1=z$, then $e=\left(1, q, a_{k}\right) \cdots$. Now $a_{1}$ is in another cycle of $e$.

$a_{k}=y$ if, and only if, $a_{1}=z$. Then $e\left(a_{1}\right)=a_{k}$ and $e\left(a_{k}\right)=a_{1}$. This shows that $e$ has order 2. However, this is only possible in case 2 . The following possibilities for $e$ remain:

(1a) $e=\left(1, q, x, \ldots, a_{1}, y, \ldots, a_{k}, z, \ldots\right) \cdots, p \geq 7$.

(1b) $e=\left(1, q, x, \ldots, a_{k}, z, \ldots, a_{1}, y, \ldots\right) \cdots, p \geq 7$.

(2a) $e=(1, q, x, \ldots)\left(a_{1}, y, \ldots\right)\left(a_{k}, z, \ldots\right) \cdots, p \geq 3$.

(2b) $e=(1, q)\left(a_{1}, y\right)\left(a_{k}, z\right) \cdots, p=2$.

Note for this that $e \in N$ and that $N$ is elementary abelian and regular. Thus all cycles in the cycle decomposition of $e$ are $p$-cycles.

For cases 1(a) and 1(b) recall that

$$
e_{1}=h \circ e \circ h^{-1}=\left(a_{1}, q, z, \ldots\right) \cdots .
$$

Since 1, $a_{1}$ and $q$ are in the same cycle of $e$ and $N$ has a unique element mapping $a_{1}$ to $q$, we see that $e_{2}=e^{i}$ for some $i$. Suppose that $q$ occurs $t$ positions after $a_{1}$ in $e$. Then $e_{1}=e^{t}$. This shows that $z$ is $t$ positions behind $q$ in $e$ and hence $a_{k}$ is also $t$ positions behind 1 in $e$. Consequently,

$$
e_{1}=\left(a_{1}, q, z, \ldots, y, x, \ldots, 1, a_{k}, \ldots\right) \quad \text { or } \quad e_{1}=\left(a_{1}, q, z, \ldots, 1, a_{k}, \ldots, y, x, \ldots\right)
$$

and $h \circ e \circ h^{-1}(1)=a_{k}$. However, also

$$
h \circ e \circ h^{-1}(1)=h \circ e\left(a_{k}\right)=h(z) .
$$

It follows that $h(z)=a_{k}$,

$$
\left(h \circ e \circ h^{-1}\right)^{-1}(x)=y
$$


and

$$
\left(h \circ e \circ h^{-1}\right)^{-1}(x)=h \circ e^{-1} \circ h^{-1}(x)=h \circ e^{-1}(y)=h\left(a_{1}\right) .
$$

Hence $h\left(a_{1}\right)=y$ and $h=\left(1, a_{1}, y, x, z, a_{k}\right)$. Thus $q=7$, i.e. $p=7$ and $f=1$.

In case 2(a) we get by Lemma 3.2(ii) that

$$
e_{1}=h \circ e \circ h^{-1}=\left(a_{1}, q, z, \ldots\right)(y, x, \ldots)\left(1, a_{k}, \ldots\right) \cdots .
$$

Hence

$$
\left(h \circ e \circ h^{-1}\right)(1)=a_{k}
$$

and

$$
\left(h \circ e \circ h^{-1}\right)(1)=(h \circ e)\left(a_{k}\right)=h(z) .
$$

It follows that $h(z)=a_{k}$. As

$$
\left(h \circ e \circ h^{-1}\right)^{-1}(x)=y
$$

and

$$
\left(h \circ e \circ h^{-1}\right)^{-1}(x)=h \circ e^{-1} \circ h^{-1}(x)=h \circ e^{-1}(y)=h\left(a_{1}\right),
$$

we get $h\left(a_{1}\right)=y$. Consequently, $h=\left(1, a_{1}, y, x, z, a_{k}\right)$. It follows that $q=7=|N|$, and so $e \in N$ consists of only one cycle. However, in case 2(a) we assumed that it has at least three cycles, and so we have a contradiction.

In the case $p \geq 3$ we considered all possibilities for how the numbers $1, a_{1}, a_{k}$ and $q$ could be ordered within $e$. There are no doubly transitive automorphism groups of a combinatorial surface of degree $q=p^{f}$ with $p \geq 3$ and $f>1$ and a regular normal subgroup. If $f=1$, the only possibility is $p=7$ and in this case we have seen in Section 2 that $C_{7} \cdot C_{6}$ is in fact a doubly transitive automorphism group of a combinatorial surface. A direct inspection shows that there is no other such group of degree 7 .

It remains to consider case 2 (b), i.e. $q=2^{f}$. In this case even more holds.

Proposition 3.3. The only primitive automorphism group of a combinatorial surface of degree $2^{f}$ with a regular normal subgroup $N$ is the symmetric group $S_{4}$.

Proof. $\quad N$ is the minimal abelian normal subgroup of $G$ [4, II, 3.2]. We may regard $G$ as a subgroup of the group of all affine transformations of the affine space $V=V(f, 2)$ of dimension $f$ over $F_{2}$ and $N$ consists of all translations, for the details we refer to 3.5 in Chapter II of [4]. So $N \cong(V,+) \cong C_{2}^{f},|N|=2^{f}$ and $N=\left\langle t_{1}, \ldots, t_{f}\right\rangle$ with $t_{i}^{2}=i d$ and $t_{i} t_{j}=t_{j} t_{i}, 1 \leq i, j \leq f$.

We show that link(1) is a 3 -cycle. For this consider the orbit of the triangle $\{1, a, b\}$ with $a, b \in\left\{2, \ldots, 2^{f}\right\}$. For each $i \in\left\{2, \ldots, 2^{n}\right\}$ there is an element $e_{i} \in N$ with $e_{i}=(1, i) \cdots$ because $N$ is regular. Thus

$$
\begin{array}{ll}
\exists e_{a} \in N: & e_{a}=(1, a)(b, u) \cdots \quad \text { and } \quad e_{a}(\{1, a, b\})=\{1, a, u\}, \\
\exists e_{b} \in N: & e_{b}=(1, b)(a, v) \ldots \quad \text { and } \quad e_{b}(\{1, a, b\})=\{1, b, v\} .
\end{array}
$$


Certainly $e_{a} \circ e_{b} \in N$ and $e_{a} \circ e_{b}=(1, x, \ldots) \cdots$. Now $N$ is abelian. Thus

$$
\left(e_{a} \circ e_{b}\right)(1)=u=\left(e_{b} \circ e_{a}\right)(1)
$$

and

$$
u=\left(e_{b} \circ e_{a}\right)(1)=e_{b}(a)=v .
$$

It follows that $u=v$. This means in the combinatorial surface there is a cycle $(a, b, u)$ and

$$
\operatorname{link}(1)=\{a, b, u,\{a, b\},\{a, u\},\{b, u\}\} .
$$

Therefore

$$
\begin{aligned}
S= & \{1, a, b, u,\{1, a\},\{1, b\},\{1, u\},\{a, b\},\{a, u\},\{b, u\},\{1, a, b\},\{1, a, u\}, \\
& \{1, b, u\},\{a, b, u\}\}
\end{aligned}
$$

and the surface $S$ consists of four vertices. A direct inspection shows that $G=S_{4}$ is the only possibility.

Proposition 3.3 completes the proof of Proposition 3.1.

\section{The General Case}

In this section we complete the proof of the theorem.

Example 4.1. There exists a doubly transitive automorphism group of a combinatorial surface without a regular normal subgroup. It is the alternating group $A_{5}$ in degree $n=6$, i.e. $G:=\langle(1,2,3,4,6),(1,4)(5,6)\rangle$ and the surface is the triangulation of the projective plane with six vertices:

$$
\begin{aligned}
S:= & \{1,2,3,4,5,6,\{1,2\},\{1,3\},\{1,4\},\{1,5\},\{1,6\}, \\
& \{2,3\},\{2,4\},\{2,5\},\{2,6\},\{3,4\},\{3,5\},\{3,6\},\{4,5\},\{4,6\},\{5,6\}, \\
& \{1,2,4\},\{1,2,5\},\{1,3,4\},\{1,3,6\},\{1,5,6\},\{2,3,6\},\{2,3,5\}, \\
& \{2,4,6\},\{3,4,5\},\{4,5,6\}\} .
\end{aligned}
$$

Proposition 4.2. $G=A_{5}$ in degree 6 is the only doubly transitive automorphism group of a combinatorial surface with no regular normal subgroup.

Proof. Let $S$ be a combinatorial surface with the doubly transitive automorphism group $G$ with no regular normal subgroup. By Lemmas 2.11 and 2.9 we know that $S$ is regular and $n-1$ is odd. Moreover, $G$ has to be a Zassenhaus group of even degree. These groups are completely classified [5, XI], [6], [2], [11]. It follows that

$$
|G|=\left(p^{f}-1\right) p^{f}\left(p^{f}+1\right) \quad \text { or } \quad|G|=\left(p^{f}-1\right) p^{f}\left(p^{f}+1\right) / 2,
$$




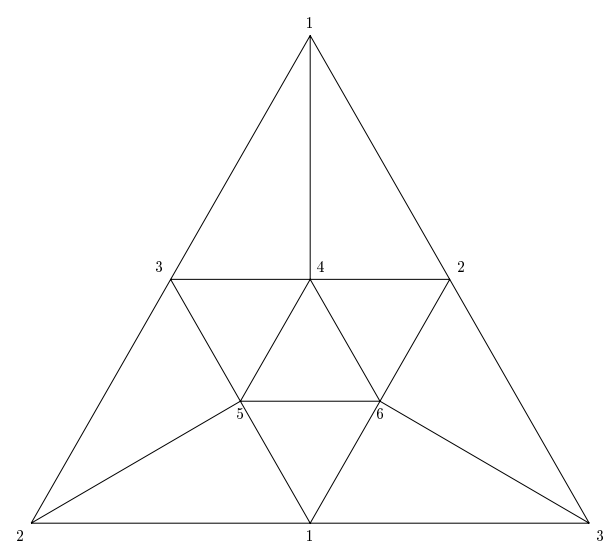

Fig. 2. The six-vertex triangulation of the real projective plane, $\{3,5\}_{5}$.

with $n=p^{f}+1$. On the other hand, we know that $|G|=2 \cdot n \cdot(n-1)$. Thus

$$
p^{f}=3 \text { or } \quad p^{f}=5 .
$$

The case $p^{f}=3$ leads to the symmetric group $S_{4}$ which is not a Zassenhaus group. Thus the degree of $G$ is six and a direct inspection shows that $A_{5}$ is the only such group. Example 4.1 shows that $A_{5}$ occurs as a doubly transitive automorphism group of a combinatorial surface.

Proposition 4.2 completes the proof of the theorem stated in Section 1. Note that a direct inspection of the combinatorial surfaces with four, six and seven vertices shows that the surface is determined up to isomorphism by its automorphism group.

Remark 4.3. (a) Edge transitive automorphism groups of combinatorial surfaces with few vertices are in some sense very close to doubly transitive groups. Using the computer algebra system GAP [3] all such groups have been determined provided the surface has no more than 23 vertices [7].

(b) These computational results show also that the groups $S_{4}, A_{5}$ and $C_{7} \cdot C_{6}$ are the only doubly transitive ones of the degrees 4,6 and 7 and that there is up to isomorphism only one combinatorial surface for these groups. Note that these facts may be also extracted from the calculations done by Lutz [9].

(c) The simple Suzuki groups are Zassenhaus groups of odd degree. Note that Lemma 2.5(iv) already shows that they cannot occur because 3 does not divide the order of a simple Suzuki group.

\section{References}

1. H. S. M. Coxeter and W. O. J. Moser, Generators and Relations for Discrete Groups, 2nd edn., Ergebnisse der Mathematik und ihrer Grenzgebiete 14, Springer-Verlag, Berlin, 1965.

2. W. Feit, On a class of doubly transitive permutation groups, Illinois J. Math. 4 (1960), 170-186. 
3. GAP4, The GAP-Group, GAP-Groups, Algorithms and Programming, Version 4.2; 2000, \{http://www. gap-system.org\}.

4. B. Huppert, Endliche Gruppen I, Grundlehren der mathematischen Wissenschaften 134, Springer-Verlag, Berlin, 1967.

5. B. Huppert and N. Blackburn, Finite Groups III, Grundlehren der mathematischen Wissenschaften 243, Springer-Verlag, Berlin, 1982.

6. N. Ito, On a class of doubly transitive permutation groups, Illinois J. Math. 6 (1962), 341-352.

7. E. Kouzoudi, 2-fach transitive Automorphismengruppen kombinatorischer Flächen, Diplomarbeit, Universität Stuttgart, 2000.

8. W. Kühnel and G. Lassmann, Neighbourly combinatorial 3-manifolds with dihedral automorphism group, Israel J. Math. 52 (1985), 147-166.

9. F. Lutz, Triangulated Manifolds with Few Vertices and Vertex-Transitive Group Actions, Thesis, TU Berlin, 1999, Shaker Verlag, Aachen, 1999.

10. P. McMullen and E. Schulte, Abstract Regular Polytopes, Cambridge University Press, Cambridge, to appear.

11. H. Zassenhaus, Kennzeichnung endlicher linearer Gruppen als Permutationsgruppen, Abh. Math. Sem. Univ. Hamburg 11 (1936), 17-44.

Received November 28, 2001, and in revised form March 19, 2002. Online publication December 20, 2002. 